\title{
Correlations in Two-Dimensional Vortex Liquids
}

\author{
Jun $\mathrm{Hu}$ and A. H. MacDonald \\ Department of Physics, Indiana University, Bloomington, IN 47405
}

(August 19, 2018)

\begin{abstract}
We report on a high temperature perturbation expansion study of the superfluid-density spatial correlation function of a Ginzburg-Landau-model superconducting film in a magnetic field. We have derived a closed form which expresses the contribution to the correlation function from each graph of the perturbation theory in terms of the number of Euler paths around appropriate subgraphs. We have enumerated all graphs appearing out to 10-th order in the expansion and have evaluated their contributions to the correlation function. Low temperature correlation functions, obtained using Padé approximants, are in good agreement with Monte Carlo simulation results and show that the vortex-liquid becomes strongly correlated at temperatures well above the vortex solidification temperature.
\end{abstract}

PACS numbers: 74.40.+k, 74.60.-w

Typeset using REVTEX 


\section{INTRODUCTION}

Because of the combination of high transition temperatures, strong anisotropy, and short coherence lengths which occurs in high temperature superconductors, strong thermal fluctuations are present over a wide temperature interval in these materials. Thermal fluctuations are especially important in a magnetic field where they are responsible for the melting of the Abrikosov 1 vortex lattice at temperatures below the mean-field critical temperature giving rise to a vortex liquid statd2. In this paper we report on a study of correlations in the vortex liquid in the extreme anisotropy limit of decoupled layers. We have evaluated the leading terms in the high temperature perturbation expansion of the superfluid density spatial correlation function for the Ginzburg-Landau model of a superconductor film.

The thermodynamics of this system is unusual because of Landau quantization of the order parameter fluctuations B; our calculations are carried out within the lowest Landau level approximation in which only the lowest gradient energy fluctuations are retained. This approximation is valid near the mean-field transition temperature and is ordinarily valid throughout the vortex liquid state, although fluctuation effects may be strong enough in some high temperature superconductors to drive the solidification transition outside of its range of validity. High-temperature perturbation expansions for the free energy of this model, even when evaluated to high order咆, exhibit little evidence of the Abrikosov vortex lattice state which is expected to occur at low temperatures. Recent Monte-Carlo simulations 6 , on the other hand, generally obtain results indicative of a weak first order phase transition between the two states. (See however Ref. [9] where a different conclusion is reached.) We find that the perturbation expansion for the superfluid density correlation function, unlike that for the average superfluid density studied by previous workers 1 , strong correlations in the vortex liquid which presage the appearance of an ordered state at low temperatures.

The paper is organized as following. In Section II we introduce the quantity we study, $\left\langle|\Delta(\vec{q})|^{2}\right\rangle$ which is proportional to the Fourier transform of the superfluid-density spatial 
correlation function. In Section III we outline the high temperature perturbation expansion for this quantity and present our closed form result for the contribution to it from individual diagrams which appear in the expansion. In Section IV we discuss our evaluation of all terms out to tenth order in this expansion and discuss some comparisons with expansions for the free energy performed by earlier workers which allow us to check our results. Extrapolations of our finite order results to low temperatures using Padé approximants are presented and compared with Monte Carlo simulations. Section V contains a brief summary.

\section{SUPERFLUID DENSITY CORRELATION FUNCTION}

The free energy per unit area of the Ginzburg-Landau model superconducting film in a perpendicular magnetic field is given by

$$
f[\Psi]=\alpha(T)|\Psi|^{2}+\frac{\beta}{2}|\Psi|^{4}+\frac{1}{2 m}|(-i \hbar \nabla-2 e \vec{A}) \Psi|^{2}
$$

where $\Psi(\vec{r})$ is the order parameter. $\Psi(\vec{r})$ is proportional to the wavefunction for the center of mass of the Cooper pairs 10 and $\vec{B}=\nabla \times \vec{A}$. (We employ the Landau gauge so that $\vec{A}=(0, B x, 0)$.) The quadratic terms in Eq. (何) are minimized by order-parameters which correspond to a lowest Landau level (LLL) wavefunction for the Cooper pairs. In using Eq. (11) we are neglecting screening so that $\vec{B}$ is the external magnetic field. This approximation is valid for type-II superconductors near the upper critical field, the regime of interest in this article. The mean-field-theory superconducting instability occurs when the quadratic terms in Eq. (可) become negative for the LLL, i.e. at $T_{c}^{M F}$ where $\alpha_{H}\left(T_{c}^{M F}\right)=0$. $\left(\alpha_{H} \equiv \alpha+\hbar e B / m^{*}\right.$.) In the LLL approximation we assume that fluctuations in higher Landau level channels can be neglected or at least absorbed in a renormalization of $\alpha(T)$ 目611. 1 . Then the order parameter $\Psi(\vec{r})$ can be expanded as

$$
\Psi(\vec{r})=\sum_{p} C_{p}\left(L_{y}\right)^{-1 / 2}\left(\frac{2 e B}{\pi \hbar}\right)^{1 / 4} e^{i p y} e^{-\frac{e B}{\hbar}\left(x-\frac{\hbar p}{2 e B}\right)^{2}}
$$

where the number of terms in the sum over $p$ is $N_{\phi}=L_{x} L_{y} / 2 \pi \ell^{2}=L_{x} L_{y}(e B / \pi \hbar)$. This expansion leads to the following expression for the Ginzburg-Landau model free energy: 


$$
\begin{gathered}
\int f[\Psi] d \vec{r}=\left\{\alpha_{H} \sum_{p}\left|C_{p}\right|^{2}+\frac{\beta}{2} \sum_{p_{1} p_{2} p_{3} p_{4}}\left(L_{y}\right)^{-1}\left(2 \pi \ell^{2}\right)^{-1 / 2} \exp \left\{-\frac{\ell^{2}}{2}\left[\sum_{i=1}^{4} p_{i}^{2}-\frac{1}{4}\left(\sum_{i=1}^{4} p_{i}\right)^{2}\right]\right\}\right. \\
\left.\times \bar{C}_{p_{1}} \bar{C}_{p_{2}} C_{p_{3}} C_{p_{4}} \delta_{p_{1}+p_{2}, p_{3}+p_{4}}\right\}
\end{gathered}
$$

where $\alpha_{H}=\alpha(T)\left(1-H / H_{c 2}(T)\right)$. Fluctuation effects in the model are regulated by the dimensionless parameter, $g \equiv \alpha_{H}\left(\pi \ell^{2} / \beta k_{B} T\right)^{1 / 2}$.

The central quantity in our work is the superfluid-density spatial correlation function which we define by

$$
\chi_{S F D}(\vec{R}) \equiv\left\langle|\Psi(\vec{r})|^{2}|\Psi(\vec{r}+\vec{R})|^{2}\right\rangle-\left\langle|\Psi(\vec{r})|^{2}\right\rangle\left\langle|\Psi(\vec{r}+\vec{R})|^{2}\right\rangle
$$

Translational invariance of the system guarantees that the right hand side of Eq. (四) is independent of $\vec{r}$. The modulation of the average superfluid density when the homogeneous system is disturbed by weak pinning can be expressed in terms of $\chi_{S F D}(\vec{R})$ :

$$
\delta\left\langle|\Psi(\vec{r})|^{2}\right\rangle=\frac{-1}{k_{B} T} \int d \vec{r}^{\prime} \chi_{S F D}\left(\vec{r}^{\prime}-\vec{r}\right) \delta \alpha\left(\vec{r}^{\prime}\right)
$$

where $\delta \alpha\left(\vec{r}^{\prime}\right)$ reflects the modulation of the mean-field transition temperature by pinning. The Fourier transform of $\chi_{S F D}(\vec{r})$ is

$$
\chi_{S F D}(\vec{q}) \equiv \frac{1}{L_{x} L_{y}} \int d^{2} \vec{r} \int d^{2} \vec{r}^{\prime}\left[\left\langle|\Psi(\vec{r})|^{2}\right| \Psi\left(\left.\vec{r}^{\prime}\right|^{2}\right\rangle-\langle| \Psi\left(\left.\vec{r}\right|^{2}\right\rangle\left\langle\left|\Psi\left(\vec{r}^{\prime}\right)\right|^{2}\right\rangle\right] \exp \left[i \vec{q} \cdot\left(\vec{r}-\vec{r}^{\prime}\right)\right]
$$

We evaluate $\chi_{S F D}(\vec{q})$ by expressing it in terms of

$$
\Delta(\vec{q}) \equiv \frac{1}{N_{\phi}} \sum_{p_{1} p_{2}} \bar{C}_{p_{1}} C_{p_{2}} \delta_{p_{2}, p_{1}+q_{y}} \exp \left[-i \ell^{2} q_{x}\left(p_{1}+p_{2}\right) / 2\right]
$$

so that

$$
\chi_{S F D}(\vec{q})=\frac{N_{\phi}^{2}}{L_{x} L_{y}}\left[\exp \left[-q^{2} \ell^{2} / 2\right]\left\langle|\Delta(\vec{q})|^{2}\right\rangle-\langle\Delta(\vec{q}=0)\rangle^{2}\right]
$$

$\Delta(\vec{q})$ satisfies the following informative sum rule 8 for each configuration of the GinzburgLandau system,

$$
\frac{1}{N_{\phi}} \sum_{\vec{q}}\left[|\tilde{\Delta}(\vec{q})|^{2}-1 / N_{\phi}\right]=0
$$


where $\tilde{\Delta}(\vec{q}) \equiv \Delta(\vec{q}) / \Delta_{0}$ and $\Delta_{0} \equiv \Delta(\vec{q}=0)$ is proportional to the integrated superfluid density 13 . (Note that $\tilde{\Delta}(\vec{q})$ is invariant when the order parameter is multiplied by an overall constant.) $\left\langle|\tilde{\Delta}(\vec{q})|^{2}\right\rangle$ is a particularly revealing quantity to examine in studying correlations in the vortex liquid. Eq. (9) guarantees that (for large $N_{\phi}$ ) $\lim _{q \rightarrow \infty} N_{\phi}|\tilde{\Delta}(\vec{q})|^{2}=1$ for any vortex liquid configuration. For example, in the high temperature (vortex gas) limit $\left\langle|\tilde{\Delta}(\vec{q})|^{2}\right\rangle=1 / N_{\phi}$ for all $\vec{q} \neq 0$. On the other hand it is easy to show that in the low temperature limit the mean-field Abrikosov lattice configuration of the order parameter gives

$$
|\tilde{\Delta}(\vec{q})|^{2}=\left\{\begin{array}{cc}
1 & \text { at } \vec{q}=\vec{G}, \\
0 & \text { otherwise. }
\end{array}\right\}
$$

where $G$ is any reciprocal lattice vector. Thus $N_{\phi}\left\langle|\tilde{\Delta}(\vec{q})|^{2}\right\rangle \equiv s_{V}(q)$ shows exactly the behavior which would be expected for the static structure function of a classical fluid with $N_{\phi}$ particles in both low temperature (solid) and high temperature (gas) limits. It seems clear that $s_{V}(q)$ must be closely related to the static structure factor of the zeroes of the order parameter, the vortices, although we do not believe that they are identical at all temperatures. For the purposes of the present study it is sufficient to observe that $h_{V}(q) \equiv$ $s_{V}(q)-1$, which we call the vortex correlation function, is a convenient measure of of the degree of correlation in this system.

\section{HIGH TEMPERATURE PERTURBATION EXPANSION}

$|\Delta(\vec{q})|^{2}$ can be expressed in the form

$$
|\Delta(\vec{q})|^{2}=N_{\phi}^{-2} \sum_{p_{1} p_{2} p_{3} p_{4}} \bar{C}_{p_{1}} \bar{C}_{p_{2}} C_{p_{3}} C_{p_{4}} \delta_{p_{1}+p_{2}, p_{3}+p_{4}}\left(\delta_{p_{1}, p_{4}+q_{y}} e^{i \ell^{2} q_{x}\left(p_{4}-p_{2}\right)}\right)
$$

At high temperatures we can evaluate its thermal average

$$
\left\langle|\Delta(\vec{q})|^{2}\right\rangle \equiv \frac{1}{Z} \int \prod_{p} d \bar{C}_{p} d C_{p}|\Delta(\vec{q})|^{2} e^{-\frac{\alpha_{H}}{K_{B} T} \int d \vec{r}|\Psi|^{2}} \times e^{-\frac{\beta}{2 K_{B} T} \int d \vec{r}|\Psi|^{4}}
$$

where $Z$ is the partition function 


$$
Z=\int \prod_{p} d \bar{C}_{p} d C_{p} e^{-\frac{\alpha_{H}}{K_{B} T} \int d \vec{r}|\Psi|^{2}} \times e^{-\frac{\beta}{2 K_{B} T} \int d \vec{r}|\Psi|^{4}}
$$

by expanding the contribution to thermal weighting factors from the quartic contribution to the Landau-Ginzburg free energy. The perturbation series can most easily be handled in terms of Feynman diagrams $\$$ 国. At $n$-th order, there are contributions from diagrams with $(n+1)$ vertices and $2(n+1)$ edges in which the edges represent the Gaussian approximation correlation functions, $n$ vertices represent $|\Psi|^{4}$ terms proportional to $\beta$ and the additional 'external' vertex corresponds to $|\Delta(\vec{q})|^{2}$. An important consequence of the Landau quantization of order parameter fluctuations is the fact that the Gaussian approximation correlation functions,

$$
\left\langle\bar{C}_{p^{\prime}} C_{p}\right\rangle=\delta_{p^{\prime}, p} k_{B} T / \alpha_{H}
$$

are independent of the momentum $p$. Each vertex has two directed outgoing lines to represent the factors of $C_{p}$ associated with it and two incoming lines to represent the factors of $\bar{C}_{p}$. All diagrams containing single-loop dressings of edges or vertices (except the external vertex) can be eliminated by following Ruggeri and Thouless 4 and expanding in terms of 'HartreeFock' approximation correlation functions. This renormalization replacest $\alpha_{H}$ in Eq. (14) by $\tilde{\alpha} \equiv-2 \alpha_{H} /\left(g^{2} \pm\left[g^{4}+4 g^{2}\right]^{1 / 2}\right)$. (The $+(-) \operatorname{sign}$ applies for $g<0(g>0)$.) $\tilde{\alpha}$ remains positive for all temperatures so that the renormalized expansion parameter of the high-temperature perturbation expansion, $x \equiv\left(\beta k_{B} T\right) /\left(4 \pi \ell^{2} \tilde{\alpha}^{2}\right)$, remains finite at all temperatures. $\left(\tilde{\alpha} \approx \alpha_{H}\right.$ for $g \gg 0, \tilde{\alpha} \approx\left|\alpha_{H}\right| / g^{2}$ for $g \ll 0 ; \tilde{\alpha}=\alpha_{H}(1-4 x)^{-1}, g^{2}=(4 x-1)^{2} / 4 x$ and $x=1 / 4$ at $T_{c}^{M F}$.) When the expansion is performed in terms of the Hartree-Fock correlation functions $\left\langle|\Delta(\vec{q})|^{2}\right\rangle$ is the sum of all the connected diagrams without single-loop dressings except at the external vertex.

It is convenient to label the external vertex as vertex 1 and to label the incoming momenta at vertex $i$ as $p_{2 i}$ and $p_{2 i-1}$. Then the contribution to $\left\langle|\Delta(\vec{q})|^{2}\right\rangle$ from an $n$-th order diagram is given by $\left(k_{B} T / \tilde{\alpha}\right)^{2}(-x)^{n} N_{\phi}^{-1} I(\vec{q}) / n$ ! where

$$
I(\vec{q}) \equiv e^{-i l^{2} q_{y}\left(q_{x}+i q_{y}\right)}\left(\frac{2 \pi}{\ell^{2}}\right)^{-n / 2} \sum_{p_{2}} \int d p_{3} \ldots \int d p_{2 n+2} \prod_{\mu=2}^{n+1} \delta\left(\sum_{i}\left(M_{\mu i}-N_{\mu i}\right) p_{i}\right)
$$




$$
\times\left(\delta_{p_{1}^{\prime}+q_{y}}+\delta_{p_{2}^{\prime}+q_{y}}\right) e^{-i \ell^{2}\left(q_{x}+i q_{y}\right) p_{2}} \exp \left\{-\frac{\ell^{2}}{2} \sum_{\mu=2}^{n+1}\left(p_{2 \mu}-p_{2 \mu-1}\right)^{2}\right\},
$$

$p_{1}^{\prime}$ and $p_{2}^{\prime}$ are the two outgoing momentum labels at vertex $1, \mu$ labels the vertices, and $M_{\mu i}$ is unity if $i$ goes into the vertex $\mu$ and is zero otherwise, while $N_{\mu i}$ is unity if $i$ comes out of $\mu$ and is zero otherwise. To obtain this result we have noted that the integral is invariant under a shift of all momenta, set $p_{1}=0$ and multiplied by $N_{\phi}$. It turns out that the integral $I(\vec{q})$ can be evaluated exactly and expressed in terms of the number of Euler paths in the two subgraphs obtained by deleting the external vertex and making the possible contractions. The two contractions correspond to the two delta functions which fix either $p_{1}^{\prime}$ or $p_{2}^{\prime}$ in Eq. (15). We denote the Euler path numbers for the two subgraphs by $T^{A}$ and $T^{B}$. The following result is derived in the Appendix:

$$
I(\vec{q})=\left\{\frac{1}{T^{A}} \exp \left(-\frac{T^{B}}{2 T^{A}} q^{2} \ell^{2}\right)+\frac{1}{T^{B}} \exp \left(-\frac{T^{A}}{2 T^{B}} q^{2} \ell^{2}\right)\right\}
$$

if $T^{A} \times T^{B} \neq 0$, and

$$
I(\vec{q})=\left(N_{\phi} \delta_{\vec{q}, 0}+1\right) /\left(T^{A}+T^{B}\right)
$$

if $T^{A} \times T^{B}=0$.

Using the above result for $I(\vec{q})$ and writing the number of appearances of a given graph as $4^{n+1} n ! / G_{n+1, g}$ we obtain the following formally exact expression for $\left\langle|\Delta(\vec{q})|^{2}\right\rangle$ :

$$
\begin{aligned}
\left\langle|\Delta(\vec{q})|^{2}\right\rangle= & \frac{1}{N_{\phi}}\left(\frac{k_{B} T}{\tilde{\alpha}}\right)^{2} \sum_{n=0}^{\infty} \sum_{g} a_{n, g}(q)(-4 x)^{n} \\
= & \frac{1}{N_{\phi}}\left(\frac{k_{B} T}{\tilde{\alpha}}\right)^{2}\left(1+N_{\phi} \delta_{\vec{q}, 0}-4 x \exp \left[-\frac{q^{2} \ell^{2}}{2}\right]+4 x^{2} \exp \left[-\frac{q^{2} \ell^{2}}{2}\right]\right. \\
& \left.+8 x^{2}\left(1+N_{\phi} \delta_{\vec{q}, 0}\right)+16 x^{2}\left(0.5 \exp \left[-\frac{q^{2} \ell^{2}}{4}\right]+\exp \left[-q^{2} \ell^{2}\right]\right)+\ldots\right),
\end{aligned}
$$

where $g$ labels graphs and the sum at $n$-th order is over $(n+1)$-vertex graphs. $a_{n, g}(q)$ is given by

$$
a_{n, g}(q)= \begin{cases}\frac{2}{G_{n+1, g}}\left(\frac{1}{T_{n, g}^{A}} \exp \left[-\frac{T_{n, g}^{B}}{2 T_{n, g}^{A}} q^{2} \ell^{2}\right]+\frac{1}{T_{n, g}^{B}} \exp \left[-\frac{T_{n, g}^{A}}{2 T_{n, g}^{B}} q^{2} \ell^{2}\right]\right) & \text { If } T_{n, g}^{A} \times T_{n, g}^{B} \neq 0, \\ \frac{2}{G_{n+1, g}\left(T_{n, g}^{A}+T_{n, g}^{B}\right)}\left(1+N_{\phi} \delta_{\vec{q}, 0}\right) & \text { If } T_{n, g}^{A} \times T_{n, g}^{B}=0,\end{cases}
$$


where $T_{n, g}^{A}$ and $T_{n, g}^{B}$ are the number of the Euler paths of the two contracted $n$-vertex graphs and $G_{n+1, g}$ is the number of automorphisms of the $(n+1)$-vertex graph with one external vertex. The diagrams which appear up to second order in the series and their associated properties are listed in Table $\square$. The explicit expression in Eq. (18) can be confirmed from the entries in this Table.

We observe in Eq. (19) that contributions which survive to the large $|\vec{q}|$ limit come only from graphs where $T_{n, g}^{A}$ equals zero or $T_{n, g}^{B}$ equals zero and that the only terms in $\left\langle|\Delta(\vec{q})|^{2}\right\rangle$ which are independent of $N_{\phi}$ come from the same set of diagrams. The contribution independent of $N_{\phi}$ is $\left\langle\Delta_{0}^{2}\right\rangle \delta_{\vec{q}, 0}$. The remaining contributions to $\left\langle|\Delta(\vec{q})|^{2}\right\rangle$, which are proportional to $N_{\phi}^{-1}$, contribute to $\chi_{S F D}(\vec{q})$ and are due to correlations in the thermally fluctuating superfluid density. We thus obtain explicitly from the perturbation expansion that

$$
\lim _{|\vec{q}| \rightarrow \infty}\left\langle|\Delta(\vec{q})|^{2}\right\rangle=\left\langle\Delta_{0}^{2}\right\rangle / N_{\phi}
$$

This result was claimed earlier on the basis of the sum rule (Eq. (9)).

The real space correlation function is given by

$$
\begin{aligned}
\left\langle|\Psi(\vec{r})|^{2}|\Psi(\vec{r}+\vec{R})|^{2}\right\rangle & \left.=\left.\frac{1}{(2 \pi)^{2}} \int d \vec{q}|| \Delta(\vec{q})\right|^{2}\right\rangle \exp \left[-q^{2} \ell^{2} / 2\right] \exp [-i \vec{q} \cdot \vec{R}] \\
& =\left(\frac{N_{\phi}}{L_{x} L_{y}}\right)^{2}\left(\frac{k_{B} T}{\tilde{\alpha}}\right)^{2} \sum_{n=0} \sum_{g} a_{n, g}(R)(-4 x)^{n} ;
\end{aligned}
$$

where

$$
a_{n, g}(R)=\frac{2}{G_{n+1, g} T_{n+1, g}}\left(\exp \left[-\frac{T_{n, g}^{A}}{2 T_{n+1, g}}\left(\frac{R}{\ell}\right)^{2}\right]+\exp \left[-\frac{T_{n, g}^{B}}{2 T_{n+1, g}}\left(\frac{R}{\ell}\right)^{2}\right]\right) .
$$

In Eq.(23) $T_{n+1, g}=T_{n, g}^{A}+T_{n, g}^{B}$ is the number of Euler paths in the uncontracted $(n+1)$ vertex graph. We see again here that only graphs with $T_{n, g}^{A}=0$ or $T_{n, g}^{B}=0$ remain finite for $R \rightarrow \infty$ where correlations vanish. The contribution of this subset of graphs is $\left\langle|\Psi(\vec{r})|^{2}\right\rangle^{2}$. 


\section{FINITE ORDER RESULTS AND EXTRAPOLATION TO LOW TEMPERATURES}

We have written a computer program which generates all relevant graphs represented by their adjacency matrices.16 From the adjacency matrices we calculate the number of Euler paths for the subgraphs, $\left(T_{n, g}^{A}\right.$ and $\left.T_{n, g}^{B}\right)$ and from the graph generating algorithm we calculate the symmetry factor $G_{n+1, g}$. In Fig. (11), we show one of the graphs which appears at second order in the expansion, the two graphs which result from the deletion of its external vertex, and the adjacency matrices of all three graphs. (The number of Euler paths equals the determinant of the minor of matrix appropriately formed from the corresponding adjacency matrix. 16 ) In this way, we have evaluated the series exactly up to tenth order 17. We have checked our results for $\left\langle|\Delta(\vec{q})|^{2}\right\rangle$ by confirming that the sum rule Eq. (9), is satisfied and that the results for both $\left\langle\left|\Delta_{0}\right|^{2}\right\rangle$ and $\sum_{\vec{q}}|\Delta(\vec{q})|^{2} \exp \left\{-\frac{1}{2} q^{2} \ell^{2}\right\}$ are correct order by order in perturbation theory. The latter two quantities can be related to derivatives of the free energy. The free energy is given by

$$
F=-k_{B} T \ln Z=k_{B} T N_{\phi}\left(\ln \left(\tilde{\alpha} / \pi k_{B} T\right)+f_{2 D}(x)\right)
$$

where the perturbation expansion for $f_{2 D}(x)$ was first calculated by Ruggeri and Thouless 4 and the expansion was extended to order $x^{11}$ by Brezin, Fujita and Hikami. 0 Differentiating Eq. (24) once with respect to $\beta$ and find that

$$
\sum_{\vec{q}}\left\langle|\Delta(\vec{q})|^{2}\right\rangle \exp \left\{-\frac{1}{2} q^{2} l^{2}\right\}=\left(\frac{k_{B} T}{\tilde{\alpha}}\right)^{2} \times \frac{4+(1-4 x) f_{2 D}^{\prime}(x)}{1+4 x}
$$

Similarly differentiating twice with respect to $\alpha_{H}$ gives

$$
\left\langle\left|\Delta_{0}\right|^{2}\right\rangle-\left\langle\Delta_{0}\right\rangle^{2}=\frac{1}{N_{\phi}}\left(\frac{k_{B} T}{\tilde{\alpha}}\right)^{2}\left(\frac{(1-4 x)\left(1-2 x f_{2 D}^{\prime}(x)\right)}{(1+4 x)^{3}}-\frac{4 x\left(f_{2 D}^{\prime}(x)+x f_{2 D}^{\prime \prime}\right)}{(1+4 x)^{2}}\right)
$$

where $\left\langle\Delta_{0}\right\rangle=\left(k_{B} T / \tilde{\alpha}\right)\left(1-2 x f_{2 D}^{\prime}(x)\right) /(1+4 x)$.

The asymptotic high-temperature expansion of $\left\langle|\Delta(\vec{q})|^{2}\right\rangle$ can be extrapolated to low temperatures using Padé approximants to describe the $x$ dependence at each value of $q$. Comparisons between Padé approximants of the series and our Monte Carlo simulation results 8 
are given in Fig. 2 for different temperatures. $s_{V}(q)$ shows a well-defined peak for $q$ near $|\vec{G}|$, where $\vec{G}$ is a reciprocal lattice vectors of the vortex solid, Such a peak is characteristic of a strongly correlated liquid. At low temperatures the [2n, 2n] Padé approximants appear to overestimate the correlations while the $[2 \mathrm{n}+1,2 \mathrm{n}+1]$ approximants appears to underestimate the correlations. Quantitative agreement with Monte Carlo simulation results is obtained for temperatures above $T_{c}^{M F}$ and for temperatures below $T_{c}^{M F}$ with $g^{2}<5$. A continuous phase-transition to a vortex solid state at low temperatures would be indicated by the divergence of $s_{v}(q)$ at $q$ near $|\vec{G}|$ and hence by a pole in the Padé approximant to $\left\langle|\Delta(\vec{q})|^{2}\right\rangle$ along the positive real $x$ axis. It might be significant that for the [4,4] Padé approximant poles on the positive real axis appear over a finite range of $q$ near $|\vec{G}|$. In Fig. 3 we plot the $q$ dependence of the value of $x_{c}$ at which these poles occur. The highest temperature at which a pole occurs is $x=9.69$ corresponding to $g^{2}=36.8$ for $q=0.91|\vec{G}|$. This value of $g^{2}$ compares with the value $g^{2}=43.5 \pm 1.0$ at which a weak first order phase transition occurs according to the Monte Carlo calculations 8 No poles on the positive real axis for $x$ occur in the $[5,5]$ Padé but we expect that they will recur at slightly lower temperatures in the $[6,6]$ Padé. We believe that the structure in $s_{V}(q)$ is a precursor of the solidification of the vortex lattice at low temperatures. The proximity of the vortex lattice state is apparent in the perturbation theory for $s_{V}(q)$ but is hidden in the perturbation theory for the free energy because of the weakness of the thermodynamic singularity associated with the phase transition. However, accurate estimates of the transition temperature based on the perturbation expansion for $s_{V}(q)$ would require calculations to be carried out to higher order than we have found possible to date.

\section{SUMMARY AND CONCLUSIONS}

We have studied the superfluid density spatial correlation function $\left\langle|\Delta(\vec{q})|^{2}\right\rangle$ using high temperature perturbation expansions and extrapolated our results to temperatures below the mean-field transition temperature by means of Padé approximants. Good agreements 
with Monte Carlo simulation data is obtained for $g>-\sqrt{5}$. Our results demonstrate that the vortex liquid is strongly correlated below $T_{c}^{M F}$. A result argued for previously on the basis of a sum rule which related the large wavevector limit of the correlation function to the average superfluid density was obtained explicitly from the perturbation expansion. We argue that these perturbation expansion studies bespeak the phase transition to a twodimensional vortex solid that is believed to occur at lower temperatures. However, higher order calculations than we have been able to complete to date would be necessary in order to obtain estimates of the transition temperature which are competitive in accuracy and reliability with those obtained previously from Monte Carlo simulations of the same model. This work was supported by the Midwest Superconductivity Consortium through D.O.E. grant no. DE-FG-02-90ER45427. The authors are grateful to Steve Girvin, Brendan McKay and Lian Zheng for helpful interactions.

\section{APPENDIX:}

The integral to be evaluated has the form:

$$
\begin{gathered}
I(\vec{q}) \equiv e^{-i l^{2} q_{y}\left(q_{x}+i q_{y}\right)}\left(\frac{2 \pi}{\ell^{2}}\right)^{-n / 2} \sum_{p_{2}} \int d p_{3} \ldots \int d p_{2 n+2} \prod_{\mu=2}^{n+1} \delta\left(\sum_{i}\left(M_{\mu i}-N_{\mu i}\right) p_{i}\right) \\
\times\left(\delta_{p_{1}^{\prime}+q_{y}}+\delta_{p_{2}^{\prime}+q_{y}}\right) e^{-i \ell^{2}\left(q_{x}+i q_{y}\right) p_{2}} \exp \left\{-\frac{\ell^{2}}{2} \sum_{\mu=2}^{n+1}\left(p_{2 \mu}-p_{2 \mu-1}\right)^{2}\right\}
\end{gathered}
$$

Of the $(2 n+1)$ variables $p_{i}$ only $n$ are independent because of the $(n+1)$ delta functions. It is convenient国 to choose $s_{\mu}=p_{2 \mu}-p_{2 \mu-1}(\mu=2, \cdots, n+1)$ as the $n$ independent variables. We label the contributions to $I(\vec{q})$ from the two choices for the delta function at the external vertex as $I^{A}(\vec{q})$ and $I^{B}(\vec{q})$ respectively. We use this delta function to eliminate the sum over $p_{2}=s_{1}$ which can be expressed as a linear function of the independent variables. For $X=A$ or $B$

$$
s_{1}^{X}=-\alpha^{X} q_{y}+\sum_{\mu=2}^{n+1} t_{\mu}^{X} s_{\mu} .
$$

The integral over the independent variables can then be expressed in terms of these coefficients. The Jacobian for changing variables from $p_{\mu}$ to $s_{\mu>1}$ is independent of $q_{y}$; at $q_{y}=0$ 
the change of variables is identical to that required for the diagram obtained by deleting the external vertex and contracting the two outgoing edges at that vertex with the two incoming edges. (The two ways of doing the contraction correspond to $I^{A}(\vec{q})$ and $I^{B}(\vec{q})$ respectively.) Following the work of McCauley and Thouless 15 we note that the inverses of the required Jacobians equal the number of Euler path\$16 for the $n$-vertex graphs which results from the deletion of the external vertex and the two possible contractions. We denote the Euler path numbers by $T^{X}$. The integral over the $s_{\mu}$ is then elementary and we obtain

$$
I^{X}(\vec{q})=\left(e^{i \ell^{2} q_{x} q_{y}}\right)^{n_{1}^{X}}\left(e^{-\ell^{2}\left|q_{y}\right|^{2}}\right)^{n_{2}^{X}}\left(e^{-\ell^{2}\left|q_{x}\right|^{2}}\right)^{n_{3}^{X}} \frac{1}{T^{X}}
$$

where $n_{1}^{X}=-1+\alpha^{X}-\sum_{\mu=2}^{n+1}\left(t_{\mu}^{X}\right)^{2}, n_{2}^{X}=-1+\alpha^{X}-\frac{1}{2} \sum_{\mu=2}^{n+1}\left(t_{\mu}^{X}\right)^{2}$ and $n_{3}^{X}=\frac{1}{2} \sum_{\mu=2}^{n+1}\left(t_{\mu}^{X}\right)^{2}$. However, we know that $I^{X}(\vec{q})$ is real which leads to the requirement that $n_{1}^{X}=0$ and implies that $I^{X}(\vec{q})=\exp \left(-q^{2} \ell^{2}\left(\alpha^{X}-1\right) / 2\right) / T^{X}$. The value of $\alpha^{X}$ can be inferred by noting that $\sum_{q} \exp \left(-q^{2} \ell^{2} / 2\right) I^{X}(\vec{q})$ is proportional to an integral which appears in the expansion of the free energy and equals $N_{\phi} / T$ where $T$ is the number of Euler paths in the original graph before deletion. It follows that $\alpha^{X}=T / T^{X}$ and hence that

$$
I(\vec{q})=\left\{\frac{1}{T^{A}} \exp \left(-\frac{T^{B}}{2 T^{A}} q^{2} \ell^{2}\right)+\frac{1}{T^{B}} \exp \left(-\frac{T^{A}}{2 T^{B}} q^{2} \ell^{2}\right)\right\}
$$

(Note that $T^{A}+T^{B}=T$.) This analysis fails in the special case where $T^{A} \times T^{B}=0$. For that case we have $I^{X}(\vec{q})=n^{X} \delta_{\vec{q}, 0}$ or $I^{X}(\vec{q})=n^{X}$ and a similar analysis gives the corresponding $n^{X}=N_{\phi} / T$ or $n^{X}=1 / T$ and so

$$
I(\vec{q})=\left(N_{\phi} \delta_{\vec{q}, 0}+1\right) /\left(T^{A}+T^{B}\right) .
$$




\section{REFERENCES}

${ }^{1}$ A. A. Abrikosov, Zh. Eksp. Teor. Fiz. 32, 1442 (1957).

${ }^{2}$ D. R. Nelson, Phys. Rev. Lett. 60, 1415 (1988); P. L. Gammel, L. F. Schneemeyer, J. V. Wasczak, and D. J. Bishop, Phys. Rev. Lett. 61, 1666 (1988).

${ }^{3}$ P. A. Lee and S. R. Shenoy, Phys. Rev. Lett. 28, 1025 (1972).

${ }^{4}$ G. J. Ruggeri and D. J. Thouless, J. Phys. F 6, 2063 (1976).

${ }^{5}$ E. Brézin, A. Fujita, and S. Hikami, Phys. Rev. Lett. 65, 1949 (1990); S. Hikami, A. Fujita, and A. I. Larkin, Phys. Rev. B 44, 10400 (1991).

${ }^{6}$ Zlatko Tešanović and L. Xing, Phys. Rev. Lett. 67, 2729 (1991).

${ }^{7}$ Yusuke Kato and Naoto Nagaosa, Phys. Rev. B. 47, 2932 (1993).

${ }^{8}$ Jun Hu and A. H. MacDonald, Phys. Rev. Lett. 71, 432 (1993).

9 J. A. O' Neill and M. A. Moore, Phys. Rev. B 48, 374 (1993).

${ }^{10}$ A. H. MacDonald, Hiroshi Akera, and M. R. Norman, Aust. J. Phys. 46, 333 (1993).

11 The limits of validity of this approximation are thoroughly discussed by Ryuske Ikeda, preprint (1993).

${ }^{12}$ We also neglect fluctuations in the vector potential. This approximation is permitted as long as the temperature is not too far below the mean-field transition temperature or the films are thin and widely separated.

${ }^{13}$ We remark that Eq. (9) is exact even for finite-size systems with quasiperiodic boundary conditions applied to the order parameter. In that case the number of $\vec{q}$ values in the sum over wavevectors is $N_{\phi}^{2}$. Finite-size effects have to be considered carefully when terms of order $N_{\phi}^{-1}$ are retained on the left hand side of Eq. (9).

${ }^{14}$ Giorgio Parisi, Statistical Field Theory (Addison-Wesley, New York, 1988). 
${ }^{15}$ G. P. McCauley and D. J. Thouless, J. Phys. F 6, 109 (1976).

${ }^{16}$ C. W. Marshall, Applied Graph Theory, Wiley-Interscience, 1971.

17 The perturbation expansion of the Fourier transform of this function has previously been evaluated to sixth order using a different approach by Ryuske Ikeda, Tetsuo Ohmi, and Toshihiko Tsuneto, J. Phys. Soc. Jpn. 59, 1397 (1990). 


\section{TABLES}

TABLE I. All diagrams up to second order in the high-temperature perturbation expansion. The open circle in each diagram represents the external vertex. 


\section{FIGURES}

FIG. 1. Example of the adjacency matrix representation of a graph which appears at second order in the expansion and of the calculation of Euler path numbers of the graph. The graph is represented by an adjacency matrix $A$ with elements $a_{i j}$ where $a_{i j}$ is equal to the number of edges going from vertex $i$ to vertex $j$. The number of Euler paths is equal to the determinant of the minor of the matrix $B$ with elements defined by $b_{i j}=\delta_{i j} \sum_{k} a_{i k}-a_{i j}$. The open circle in the uncontracted graphs represents the external vertex. The two graphs on the left result from the two possible contractions after deletion of the external vertex. The contribution of this graph to the series is $a_{2}(q)=0.5 \exp \left(-q^{2} \ell^{2} / 4\right)+\exp \left(-q^{2} \ell^{2}\right)$.

FIG. 2. Comparisons of the perturbation calculation and Monte Carlo simulations. The $y$-axis label is $\left\langle|\tilde{\Delta}(\vec{q})|^{2}\right\rangle$, while the $x$-axis is the wave vector in the units of the inverse magnetic length $\ell^{-1}$. The upper-left panel is for a temperature above $T_{c}^{M F}$, while the other panels are for temperatures

below $T_{c}^{M F}$. The arrows in the plots indicate the location of reciprocal vector, $|\vec{G}|$, of the vortex lattice state. The Padé approximants sometimes behave poorly at small wavevectors. $(q \ell \sim 1.0$. $)$ We have 'clipped' anomalous small wavevector behavior in some of these figures so as not to obscure the behavior for $q$ near $G$.

FIG. 3. Wavevector dependence of one of the poles in the $[4,4]$ Padé approximant. The pole moves from negative to positive $x$ by passing through $\infty$. This approximant has poles for $q$ near $G$ at temperatures which are somewhat higher than the temperature where a (weakly) first order phase transition is indicated by Monte Carlo simulations. (See text.) The arrows in the plot indicate the location of reciprocal vector $|\vec{G}|$ of the vortex lattice state. 